\title{
Immediate application of vacuum assisted closure dressing over free muscle flaps in the lower extremity does not compromise flap survival and results in decreased flap thickness
}

\author{
Harvey Chim, Yasmina Zoghbi, Ajani George Nugent, Wrood Kassira, Morad Askari, \\ Christopher John Salgado \\ Division of Plastic Surgery, University of Florida College of Medicine, Gainesville, FL, USA
}

Background Free muscle flaps are a mainstay for reconstruction of distal third leg wounds and for large lower extremity wounds with exposed bone. However a major problem is the significant postoperative flap swelling, which may take months to resolve. We studied the efficacy and safety of immediate application of a vacuum assisted closure (VAC) dressing after a free muscle flap to the lower extremity.

Methods Over a 19 months period, all consecutive free muscle flaps for lower extremity reconstruction at a Level I trauma center were evaluated prospectively for postoperative flap thickness, complications and flap survival. Immediate application of a VAC dressing was performed in 9 patients, while the flap was left exposed for monitoring in 8 patients.

Results There was no statistically significant difference in flap survival between both cohorts. Mean flap thickness at postoperative day 5 for the VAC group was $6.4 \pm 6.4 \mathrm{~mm}$, while flap thickness for the exposed flap group was $29.6 \pm 13.5 \mathrm{~mm}$. Flap thickness was significantly decreased at postoperative day 5 for the VAC dressing group.

Conclusions Immediate application of VAC dressing following free muscle flaps to the lower extremity does not compromise flap survival or outcomes and results in decreased flap thickness and a better aesthetic outcome.

Keywords Free tissue flaps / Negative pressure wound therapy / Reconstructive surgery / Tibial fractures / Gustilo classification / Surgical flaps

Received: 25 Nov $2016 \bullet$ Revised: 30 Jul $2017 \bullet$ Accepted: 12 Sep 2017

pISSN: 2234-6163 • elSSN: 2234-6171 • https://doi.org/10.5999/aps.2016.01977• Arch Plast Surg 2018;45:45-50
Correspondence: Harvey Chim

Division of Plastic and Reconstructive Surgery, University of Florida College of Medicine, 1600 SW Archer Rd, PO Box 100138, Gainesville, FL 32610, USA

Tel: +1-352-273-8670

Fax: +1-352-273-8639

E-mail: harveychim@yahoo.com

\section{INTRODUCTION}

Free muscle flaps are a workhorse for reconstruction of lower extremity wounds. Established indications include distal third wounds and large defects with exposed bone, tendon or other vital structures. There remains a preference among many orthopedic surgeons, for muscle flap coverage, in the belief that this has a beneficial effect on decreasing infection rates [1]. The increased capacity of muscle compared to fasciocutaneous flaps for inhibiting bacterial growth has been shown in animal studies 
[2-4]. However, the published literature has not shown a difference between free muscle and free fasciocutaneous flaps for lower extremity coverage $[5,6]$.

A major problem with free muscle flaps in the lower extremity is postoperative swelling. This necessitates a "dangle protocol" to allow the free muscle to get used to the increased effect of gravity. Nevertheless, the swelling in the free muscle can take many months to resolve, and may never approach the contour and thickness of normal skin in the lower extremity. The muscle edema can also prolong the healing course, leading to problems such as loss of skin graft.

A solution used by the senior author and some other surgeons is immediate application of a vacuum assisted closure (VAC) dressing after free muscle coverage of the lower extremity. The VAC dressing has been shown to optimize blood flow, decrease local tissue edema and remove excess fluid from the wound bed [7]. In this fashion, the free muscle flap does not increase in thickness postoperatively, preventing the swelling normally seen following microsurgical transfer to the lower extremity. Another advantage of the VAC dressing is that regular irrigation of the flap to prevent desiccation is not required. The VAC dressing also has been shown to encourage take and survival of skin grafts over the free muscle flap [8]. Flap monitoring relies entirely on the Cook-Swartz implantable Doppler device (Cook Medical, Bloomington, IN, USA).

A criticism of this approach is that there is no way to monitor the flap using traditional techniques, such as visual observation and transcutaneous Doppler signals. While the benefits of a VAC dressing are not in question, flap monitoring is the main concern that prevents widespread adoption of this technique.

The aim of this study was to prospectively compare flap outcomes and flap thickness in a level I trauma center between consecutive patients who had immediate postoperative application of a VAC dressing following free muscle flap coverage of lower extremity wounds and patients who had the muscle exposed for monitoring.

\section{METHODS}

A prospective study was performed in a level I trauma center for all patients who underwent free muscle flap coverage for leg wounds distal to the knee. This was performed over a 19 months period from December 2014 to June 2016 involving all consecutive patients, with at least 2 months follow-up postoperatively for all patients. The institutional review board approved the study and patients gave verbal informed consent for flap measurements. The senior author performed immediate application of a VAC dressing following free muscle coverage of lower ex- tremity wounds (Group 1), while the other microsurgeons in the institution left the flap exposed for monitoring, with wet to dry dressings and regular saline irrigation (Group 2). The VAC was set on $75 \mathrm{~mm} \mathrm{Hg}$ on continuous suction. The VAC dressing when used was removed on postoperative day 5 . There were 9 free muscle flaps performed in Group 1 and 8 in Group 2, for a total of 17 consecutive flaps. A standard postoperative dangle protocol was followed, with progressive dangling commenced at postoperative day 14 .

Outcomes inclusive of flap survival and complications were recorded, in addition to demographic data. Flap thickness, defined as the distance between the highest point of the flap and the adjacent skin was measured (Fig. 1) at regular intervals on postoperative day 5 and when the patient presented to the outpatient clinic for short and long-term follow-up. Long-term follow-up was not available for all patients. The majority of patients were treated at a county hospital, with many homeless, uninsured or from out of state. Hence many were not willing to return for follow-up flap measurements. Statistical analysis was performed using the student's t-test or chi square test to compare flap thickness and other variables between groups.

\section{RESULTS}

Patient data is presented in Table 1. Mean age of patients in Group 1 was $40.6 \pm 16.1$ years, while in Group 2 mean age was $35.1 \pm 16.0$. There was no significant difference in age or body mass index between groups. All patients had Gustilo IIIB open tibial fractures of the leg that required coverage except for 3 pa-

\section{Fig. 1. Measurement of flap thickness}

Measurement of flap thickness was performed between the skin edge and the highest and thickness point of the flap. In this case, the skin edge is at $10 \mathrm{~mm}$ on the ruler and highest point of the flap at $21 \mathrm{~mm}$, hence the flap thickness was recorded as $11 \mathrm{~mm}$.

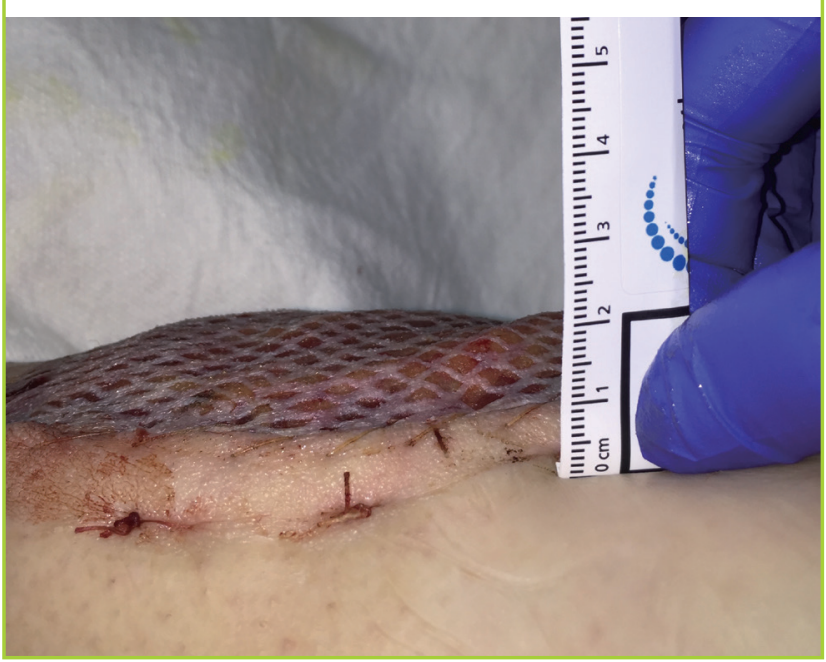


Table 1. Patient data

\begin{tabular}{|c|c|c|c|c|c|c|c|c|c|}
\hline Case & $\begin{array}{l}\text { Age } \\
\text { (yr) }\end{array}$ & Sex & $\begin{array}{c}\mathrm{BMI} \\
\left(\mathrm{kg} / \mathrm{m}^{2}\right)\end{array}$ & $\begin{array}{l}\text { Location } \\
\text { of defect }\end{array}$ & Reason for flap & $\begin{array}{c}\text { Flap size } \\
\left(\mathrm{cm}^{2}\right)\end{array}$ & $\begin{array}{l}\text { Recipient } \\
\text { vessel }\end{array}$ & $\begin{array}{c}\text { Type of arterial } \\
\text { anastomosis }\end{array}$ & $\begin{array}{l}\text { VAC } \\
(\mathrm{Y} / \mathrm{N})\end{array}$ \\
\hline 1 & 29 & Male & 31.5 & Foot & Open 1st metatarsal fracture & Latissimus & 240 & PT & ETE \\
\hline 2 & 25 & Male & 27.2 & Leg & Trauma/Gustilo 3B tibia fracture & Latissimus & 294 & AT & ETE \\
\hline 3 & 40 & Female & 33.1 & Foot & Recurrent plantar fibromatosis & Serratus & 100 & PT & ETE \\
\hline 4 & 56 & Male & 27 & Leg & Trauma/Gustilo 3B tibia fracture & Latissimus & 184 & AT & ETS \\
\hline 5 & 49 & Male & 28.3 & Leg & Trauma/Gustilo 3B tibia fracture & Latissimus & 144 & PT & ETS \\
\hline 6 & 53 & Female & 22.9 & Leg & Trauma/Gustilo 3B tibia fracture & Latissimus & 112 & PT & ETS \\
\hline 7 & 53 & Female & 38.3 & Heel & Chronic osteomyelitis & Latissimus & 144 & PT & ETE \\
\hline 8 & 34 & Male & 26 & Leg & Trauma/Gustilo 3B tibia fracture & Latissimus & 300 & PT & ETE \\
\hline 9 & 19 & Female & 18.1 & Leg & Trauma/Gustilo 3B tibia fracture & Latissimus & 161 & PT & ETE \\
\hline 10 & 58 & Male & 25.4 & Leg & Trauma Gustilo 3B tibia fracture & Latissimus & 198 & PT & ETE \\
\hline \multirow[t]{2}{*}{11} & 46 & Male & 36.2 & Leg & Chronic osteomyelitis & Gracilis & 200 & PT & ETE \\
\hline & & & & & & Latissimus & 350 & AT & ETE \\
\hline 12 & 23 & Male & 25.2 & Leg & Trauma/Gustilo 3B tibia fracture & Latissimus & 140 & PT & ETE \\
\hline 13 & 18 & Male & 21.9 & Leg & Trauma/Gustilo 3B tibia fracture & Latissimus & 350 & PT & ETE \\
\hline 14 & 25 & Male & 32.2 & Leg & Trauma/Gustilo 3B tibia fracture & Rectus & 225 & AT & ETE \\
\hline 15 & 20 & Female & 33.3 & Leg & Trauma/Gustilo 3B tibia fracture & Rectus & 300 & PT & ETE \\
\hline 16 & 63 & Male & 29.2 & Leg & Trauma/Gustilo 3B tibia fracture & Rectus & 300 & Popliteal artery & ETS \\
\hline
\end{tabular}

BMI, body mass index; VAC, vacuum assisted closure dressing; PT, posterior tibial artery; ETE, end-to-end anastomosis; AT, anterior tibial artery; ETS, end-to-side anastomosis.

\section{Table 2. Flap outcome data}

\begin{tabular}{|c|c|c|c|c|c|}
\hline Case & VAC & Flap thickness POD5 (mm) & Flap thickness (late, mm) & Flap loss & Outcome \\
\hline 1 & Y & 20 & 20 (2 mo); 0 (15 mo) & $\mathrm{N}$ & Uncomplicated \\
\hline 2 & Y & 6 & 6 (20 day); 1 (74 day) & N & Uncomplicated \\
\hline 3 & N & 40 & 35 (35 day) & N & Uncomplicated \\
\hline 4 & Y & 2 & 2 (16 day) & N & Uncomplicated \\
\hline 5 & Y & 3 & & N & Uncomplicated \\
\hline 6 & Y & 0 & 0 (10 day) & Y & Integra/split thickness skin graft/limb salvage \\
\hline 7 & Y & 11 & 10 (8 day) & N & Uncomplicated \\
\hline 8 & N & 15 & & N & Uncomplicated \\
\hline 9 & Y & 2 & 2 (10 day) & N & Uncomplicated \\
\hline 10 & Y & 3 & 3 (10 day) & N & Uncomplicated \\
\hline 11 & $\mathrm{~N}$ & 45 & & Y & Repeat flap/limb salvage \\
\hline 12 & Y & 11 & 6 (21 day) & N & Uncomplicated \\
\hline 13 & N & 12 & & N & Uncomplicated \\
\hline 14 & $\mathrm{~N}$ & 35 & & N & Uncomplicated \\
\hline 15 & $\mathrm{~N}$ & 20 & & N & Uncomplicated \\
\hline 16 & $\mathrm{~N}$ & 40 & & Y & Below knee amputation \\
\hline
\end{tabular}

tients with other pathology involving the foot and heel in Group 1 and 1 patient with chronic osteomyelitis of the tibia in Group 2. Flap outcome data is shown in Table 2. There was no statistically significant difference in flap survival between both groups. Flap thickness was measured on postoperative day 5 in all patients. Mean flap thickness at postoperative day 5 for the VAC group was $6.4 \pm 6.4 \mathrm{~mm}$, while flap thickness for the exposed flap group was $29.6 \pm 13.5 \mathrm{~mm}$. There was a statistically significant difference $(\mathrm{P}<0.05)$ in flap thickness between patients treated with a VAC dressing and those where the flap was left exposed for monitoring. Figs. 2 and 3 illustrate the difference in flap thickness and decreased swelling seen with free muscle flaps treated with an immediate VAC dressing compared to those left open to air for monitoring. Fig. 4 shows the natural evolution of a flap for coverage for distal third leg wound treated with immediate application of the VAC. Postoperative flap swelling encountered routinely with muscle flaps was not seen. The thickness of the flap remained similar on short and long-term follow-up.

In the VAC dressing group (Group 1), there was 1 flap failure due to previously undiagnosed deep vein thrombosis (diag- 
Fig. 2. Comparison between flaps with VAC and open dressings

Comparison between flaps with VAC and open dressings in the foot treated with immediate postoperative VAC dressing (Group 1; $\mathrm{A}-\mathrm{C}$ ) and wet to dry dressings (Group 2; D-F). Free latissimus dorsi flap to left foot (Group 1) at postoperative day 7 (A), 21 (B) and 4 months (C). Flap is not bulky after VAC dressing is taken down and has achieved its final thickness at 4 months postoperative. Free serratus anterior flap to left foot (Group 2) at postoperative day 14 (D), 1 month (E) and 8 months (F). Flap remains bulky in the immediate postoperative period and does not atrophy until 8 months postoperatively. VAC, vacuum assisted closure dressing.
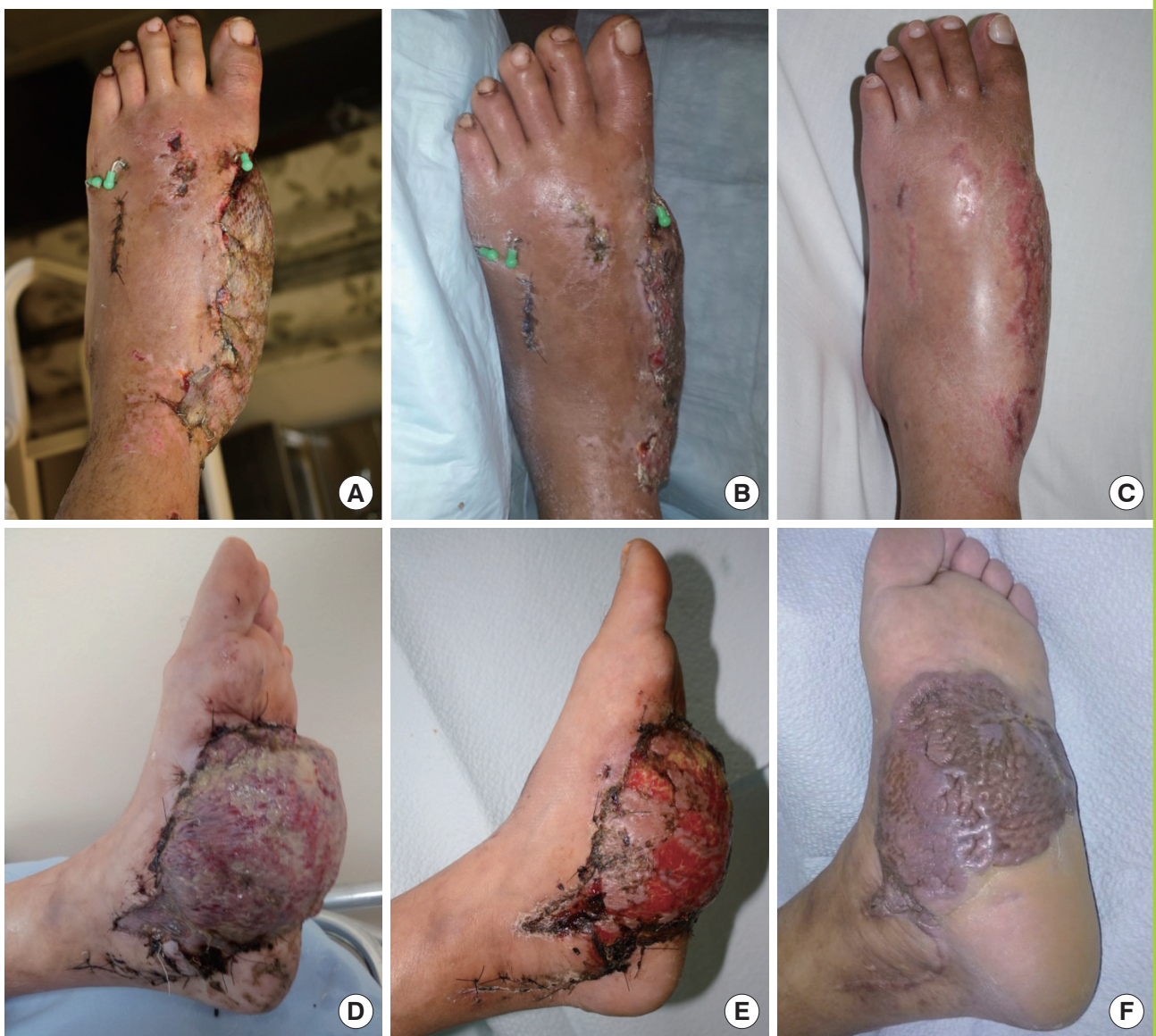

Fig. 3. Comparison between flaps with VAC and open dressings

Comparison between flaps with VAC and open dressings in the leg in the immediate postoperative period treated with VAC dressing (Group 1; $A$, B) and wet to dry dressings (Group 2; C, D). Group 1, free latissimus dorsi flaps in 2 different patients at postoperative day 6 for coverage of large anterior and medial leg wounds (A, B); Group 2, free latissimus dorsi flap at postoperative day 6 for coverage of anterior leg wound (C); free rectus flap at postoperative day 7 for coverage of anterior leg wound (D). Marked swelling is seen for the flaps in Group 2, but not in Group 1, where the flaps are almost even in thickness with the adjacent skin. VAC, vacuum assisted closure dressing.
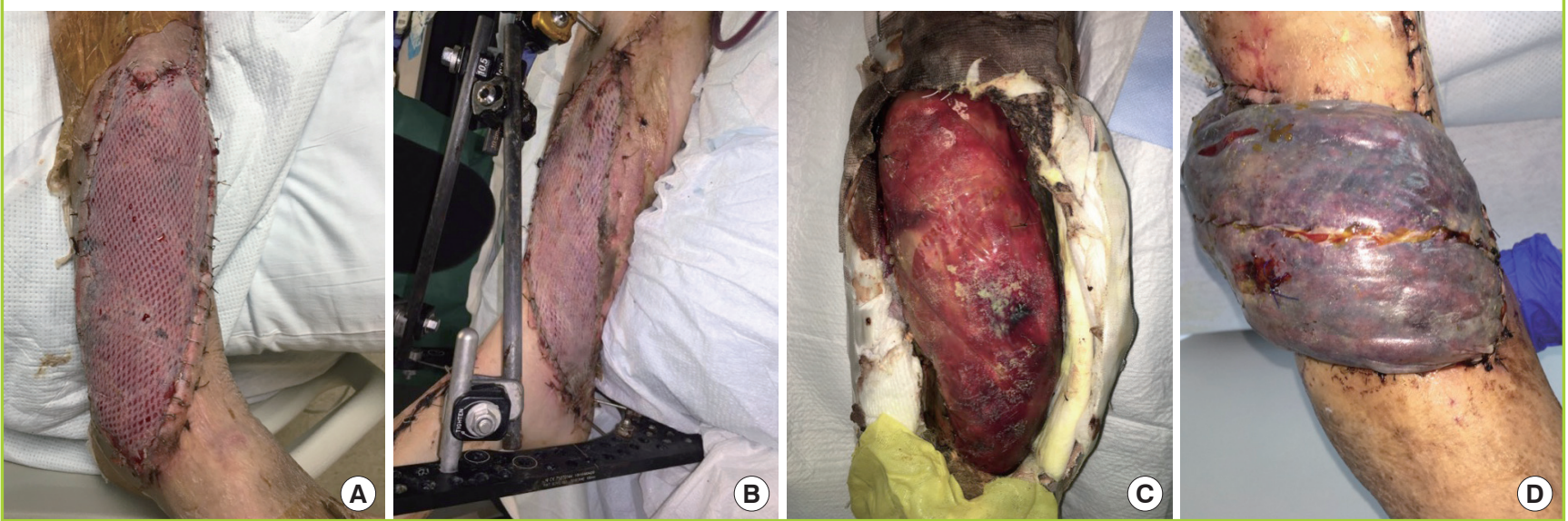

nosed after the flap was performed) involving the entire deep venous system of the affected lower extremity from the pelvis to the crural veins, leading to venous outflow obstruction. This re- sulted in delayed flap failure from venous congestion after 2 weeks. The wound was treated with Integra (Integra Lifesciences) and subsequently skin grafted, with successful limb salvage. 


\section{Fig. 4. Evolution of flap over time}

Evolution of flap appearance and thickness over time in a patient with free latissimus dorsi flap and immediate application of a VAC dressing for coverage of distal third medial leg wound. (A) Immediate postoperative, (B) postoperative day 9, (C) 6 months postoperative. Postoperative flap swelling encountered routinely with muscle flaps is not seen. The thickness of the flap remains similar on short and long-term follow-up. VAC, vacuum assisted closure dressing.

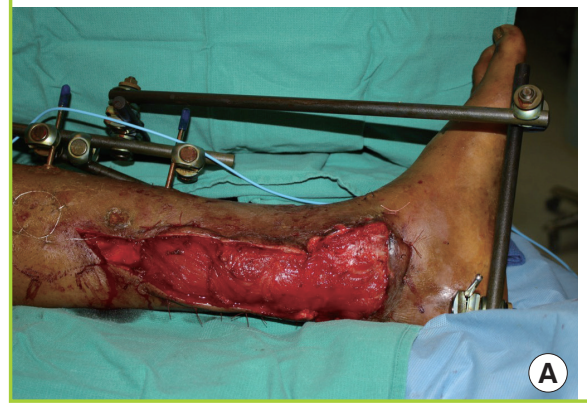

In Group 2, there were 2 flap failures. One patient had flap loss due to arterial thrombosis and was treated with a repeat muscle flap to a different recipient artery on postoperative day 7, with successful limb salvage. The other patient had flap loss due to persistent unresolved infection from antibiotic resistant Pseudomonas Aeruginosa, which resulted in a below knee amputation. No other flaps required flap exploration nor were otherwise compromised in the postoperative period.

\section{DISCUSSION}

The VAC dressing has the advantage of reducing edema of the flap in the short term. In the senior author's limited experience, this also translates to earlier resolution of edema, which is a persistent problem with free muscle flaps to the lower extremity, that can take up to 1 year to resolve. Other mechanisms of action of the VAC dressing include optimizing blood flow and removal of excess fluid from the wound bed, though these are probably less important in this application. The reduction of edema through the VAC dressing most likely occurs through microdeformation of the local wound environment by the VAC sponge allowing excess interstitial fluid to be removed from the muscle flap as well as macrodeformation which allows the VAC sponge itself to act as a splint to exert a limited amount of compression over the flap to reduce the development of edema in the immediate postoperative period.

In addition, by reducing the amount of swelling in the immediate postoperative period, the VAC dressing prevents a wrinkled appearance of the flap in the long-term following atrophy of the muscle, which results when a very swollen flap atrophies and leaves an excess skin grafted surface. Without the VAC dressing, we have found that flap revision is often necessary, due to patient requests to reduce the thickness of the flap and remove excess tissue. For reconstruction in the foot, this is partic- ularly a problem, as patients often have problems fitting adequate footwear due to thickness of the flap. With immediate application of the VAC, we have found that these problems are to a certain extent, ameliorated.

A limitation of this study is the small sample size in each cohort. However, with the increasing use of propeller and perforator based flaps and free fasciocutaneous flaps for reconstruction of distal third lower extremity wounds, it is difficult for any one center to accumulate a large number of patients requiring free muscle flaps for lower extremity reconstruction. This is true in our center, where the number of patients requiring a free muscle flap has decreased dramatically due to other available options for reconstruction. In addition a true prospective randomized trial examining the efficacy of the VAC dressing will probably be impossible to perform, because each surgeon will have his or her own preconceptions about the safety of immediate VAC dressing over free muscle flaps and flap monitoring, and will be unlikely to compromise these beliefs in a complex and high risk procedure like a free flap. Another limitation of the study is the short-term follow-up measurement of flap thickness in some patients. However, due to the nature of the patient population that sustains these types of injuries in our cohort, long-term followup was not possible in many patients. Finally three patients in the group without VAC dressing had a rectus muscle flap, which is often thicker than the latissimus, or serratus muscle flaps. However, patients who had latissimus flaps in the group without VAC dressing also had noticeably increased flap thickness.

Nevertheless, this prospective study represents the only published evidence in the literature, to our knowledge, about the efficacy and safety of the VAC dressing for immediate application following lower extremity free muscle flaps. Our data supports the immediate application of VAC dressings over free muscle flaps to the lower extremity, and shows that this technique does not compromise flap survival. 


\section{CONFLICT OF INTEREST}

No potential conflict of interest relevant to this article was reported.

\section{PATIENT CONSENT}

The patient provided written informed consent for the publication and the use of their images.

\section{REFERENCES}

1. Irons GB Jr, Wood MB. Soft-tissue coverage for the treatment of osteomyelitis of the lower part of the leg. Mayo Clin Proc 1986;61:382-7.

2. Chang N, Mathes SJ. Comparison of the effect of bacterial inoculation in musculocutaneous and random-pattern flaps. Plast Reconstr Surg 1982;70:1-10.

3. Calderon W, Chang N, Mathes SJ. Comparison of the effect of bacterial inoculation in musculocutaneous and fasciocutaneous flaps. Plast Reconstr Surg 1986;77:785-94.

4. Gosain A, Chang N, Mathes S, et al. A study of the relationship between blood flow and bacterial inoculation in musculocutaneous and fasciocutaneous flaps. Plast Reconstr Surg 1990;86:1152-62.

5. Yazar S, Lin CH, Lin YT, et al. Outcome comparison between free muscle and free fasciocutaneous flaps for reconstruction of distal third and ankle traumatic open tibial fractures. Plast Reconstr Surg 2006;117:2468-75.

6. Fox CM, Beem HM, Wiper J, et al. Muscle versus fasciocutaneous free flaps in heel reconstruction: systematic review and meta-analysis. J Reconstr Microsurg 2015;31:59-66.

7. Venturi ML, Attinger CE, Mesbahi AN, et al. Mechanisms and clinical applications of the vacuum-assisted closure (VAC) device: a review. Am J Clin Dermatol 2005;6:185-94.

8. Lambert KV, Hayes P, McCarthy M. Vacuum assisted closure: a review of development and current applications. Eur J Vasc Endovasc Surg 2005;29:219-26. 